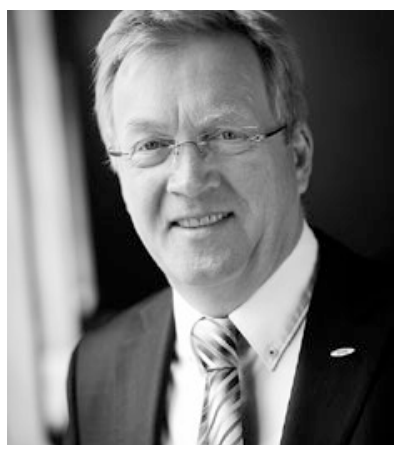

Andreas Westerfellhaus

Präsident des

Deutsches Pflegerats (DPR)
Deutscher Pflegerat e.V.

Bundesarbeitsgemeinschaft

Pflege- und Hebammenwesen

In Kooperation mit

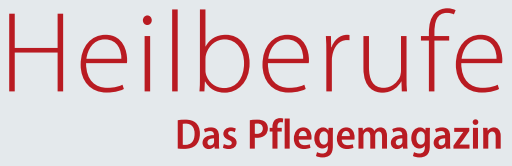

\title{
Editorial
}

\section{Neuer Pflegebedürftigkeitsbegriff braucht neue Ausbildung}

$\mathrm{D}$ eutschland wird älter und damit steigt auch die Zahl der Menschen, die an Demenz erkrankt sind. Um die dadurch an die professionell Pflegenden gestellten steigenden Herausforderungen zu meistern, ist eine neue, moderne Form der Pflegeausbildung erforderlich, wie dies das Pflegeberufereformgesetz vorsieht. Es ist unsere Pflicht, für eine bestmögliche medizinische und pflegerische Versorgung und Betreuung der Menschen zu sorgen, die unserer Hilfe bedürfen. Das kann jedoch nur gelingen, wenn wir die richtigen Rahmenbedingungen setzen. Der Gesetzgeber hat mit dem Zweiten Pflegestärkungsgesetz einen neuen Pflegebedürftigkeitsbegriff geschaffen, der ab dem 1. Januar 2017 einen veränderten $\mathrm{Zu}$ gang zu den Leistungen der Pflegeversicherung vorsieht. Im Mittelpunkt steht dabei der Grad der Selbstständigkeit. Das ist wegweisend und wird zu Verbesserungen der Versorgungssituation führen. Voraussetzung hierfür ist jedoch, dass das dazu benötigte Personal in der Menge als auch mit der richtigen Qualifikation vorhanden ist. Einer der wesentlichsten Stellhebel wird dabei das Pflegeberufereformgesetz sein. Es sichert die bestmögliche Versorgung der pflegebedürftigen Menschen, unabhängig davon ob diese im Krankenhaus oder in einer Pflegeeinrichtung gepflegt und betreut werden. Der Bundesgesetzgeber muss jetzt dafür sorgen, dass das für die Pflegefachpersonen und die Patientensicherheit unabdingbare Pflegeberufereformgesetz ohne faule Kompromisse verabschiedet wird.

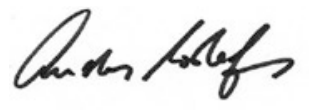

Andreas Westerfellhaus

Präsident des Deutschen Pflegerats (DPR)

\section{IM FOCUS}

\section{Ambulant völlig neu denken}

Die Ergebnisbeurteilung und Qualitätsberichterstattung in der ambulanten Pflege muss auf den Kopf gestellt werden. Hierzu hat der Gesetzgeber eine eigenständige Entwicklung für den ambulanten Bereich festgeschrieben. Was fehlt, ist der entscheidende Schritt hin zu wissenschaftlich fundierten Indikatoren für eine möglichst objektive Qualitätsmessung.

Denkt man diesen Prozess aus der Patientenperspektive heraus, wird schnell klar, dass es vielfach um subjektive Faktoren, um Empfindungen geht, zum Beispiel, ob die Pflegekräfte freundlich und hilfsbereit und die personellen Wechsel möglichst gering sind. Anders sieht der Blick aus Sicht der professionell Pflegenden aus. Diesen fällt es häufig schwer, gute Pflege zu definieren, hat jüngst Dr. Klaus Wingenfeld von der Universität Bielefeld gesagt. Das Wohlbefinden des Patienten und dessen Erhaltung bzw. Steigerung der Eigenaktivität scheinen für sie wichtige Aspekte zu sein.

Die ambulante Pflege benötigt einen Weg abseits der bürokratischen Auflagen der bisherigen Qualitätsebenen. Qualität sollte sich daraus ermessen, ob und wie klar definierte Aufgaben u.a. bei der Bewältigung von Krankheitsfolgen erfolgreich erfüllt werden und welche Folgen sich daraus ergeben, macht Wingenfeld weiter deutlich. Diese Kehrtwende im Qualitätsansatz baut auf dem neuen Pflegebedürftigkeitsbegriff auf und ist zugleich weit weg von der heutigen Realität. Erforderlich sind dafür ein neues Denken aller Beteiligten, einhergehend mit neuen fachlichen Anforderungen an die professionell Pflegenden.

\section{Thomas Meißner}

Präsidiumsmitglied des Deutschen Pflegerats (DPR) 